\title{
Genetic parameters of ovarian and uterine reproductive traits in dairy cows
}

\author{
T. R. Carthy, ${ }^{*} \dagger$ D. P. Ryan, $\ddagger$ A. M. Fitzgerald, ${ }^{*}$ R. D. Evans, $§$ and D. P. Berry ${ }^{* 1}$ \\ ${ }^{*}$ Animal \& Grassland Research and Innovation Centre, Teagasc, Moorepark, Co. Cork, Ireland \\ †School of Veterinary Medicine, University College Dublin, Belfield, Dublin 4, Ireland \\ $\ddagger$ Reprodoc Ltd., Fermoy, Co. Cork, Ireland \\ §lrish Cattle Breeding Federation, Bandon, Co. Cork, Ireland
}

\section{ABSTRACT}

The objective of the study was to estimate genetic parameters of detailed reproductive traits derived from ultrasound examination of the reproductive tract as well as their genetic correlations with traditional reproductive traits. A total of 226,141 calving and insemination records as well as 74,134 ultrasound records from Irish dairy cows were used. Traditional reproductive traits included postpartum interval to first service, conception, and next calving, as well as the interval from first to last service; number of inseminations, pregnancy rate to first service, pregnant within $42 \mathrm{~d}$ of the herd breeding season, and submission in the first $21 \mathrm{~d}$ of the herd breeding season were also available. Detailed reproductive traits included resumed cyclicity at the time of ultrasound examination, incidence of multiple ovulations, incidence of early postpartum ovulation, heat detection, ovarian cystic structures, embryo loss, and uterine score; the latter was a subjectively assessed on a scale of 1 (little fluid with normal uterine tone) to 4 (large quantity of fluid with a flaccid uterine tone). Variance (and covariance) components were estimated using repeatability animal linear mixed models. Heritability for all reproductive traits were generally low (0.001-0.05), with the exception of traits related to cyclicity postpartum, regardless if defined traditionally (0.07; calving to first service) or from ultrasound examination [resumed cyclicity at the time of examination (0.07) or early postpartum ovulation (0.10)]. The genetic correlations among the detailed reproductive traits were generally favorable. The exception was the genetic correlation (0.29) between resumed cyclicity and uterine score; superior genetic merit for cyclicity postpartum was associated with inferior uterine score. Superior genetic merit for most traditional reproductive traits was associated with superior genetic merit for resumed cyclicity (genetic correlations ranged from -0.59 to -0.36 and from 0.56 to 0.70 ) and uterine

Received October 1, 2014

Accepted February 23, 2015.

${ }^{1}$ Corresponding author: Donagh.Berry@teagasc.ie score (genetic correlations ranged from -0.47 to 0.32 and from 0.25 to 0.52 ). Genetic predisposition to an increased incidence of embryo loss was associated with both an inferior uterine score (0.24) and inferior genetic merit for traditional reproductive traits (genetic correlations ranged from -0.52 to -0.42 and from 0.33 to 0.80$)$. The results from the present study indicate that selection based on traditional reproductive traits, such as calving interval or days open, resulted in improved genetic merit of all the detailed reproductive traits evaluated in this study. Additionally, greater accuracy of selection for calving interval is expected for a relatively small progeny group size when detailed reproductive traits are included in a multitrait genetic evaluation.

Key words: fertility, genetic correlation, ultrasound, detailed reproductive trait

\section{INTRODUCTION}

Traditional measures of reproductive performance recorded by producers are routinely available and are now included in many national dairy breeding goals (Miglior et al., 2005). Traditional reproductive traits such as calving interval (CI), days open (DO), and pregnancy rate to first service (PRFS) are, however, generally not very heritable $(<0.05$; Berry et al., 2014). The observed low heritability delays the achievement of high accuracy of selection and, thus, genetic gain for reproductive traits (Berry et al., 2014).

Decomposing aggregate reproductive phenotypes into their detailed components, which are potentially less influenced by management, could prove to be more heritable and, assuming sufficient genetic variation exists, genetic gain for reproductive performance could be accelerated. For example, CI is composed of several detailed reproductive components, such as the postpartum interval to commencement of estrus cyclicity, expression of estrus, conception, maintenance of pregnancy, and gestation length. Therefore, a cow with a long calving to first service interval (CFS) but a good pregnancy rate may have a similar phenotypic value for CI to a cow with a short CFS but poor pregnancy rate. 
These are profoundly distinct cows, yet have the same phenotypic value, and potentially the same estimated breeding value for CI if estimated in a univariate genetic evaluation model.

Detailed reproductive traits, such as postpartum commencement of luteal activity measured by progesterone levels, are both phenotypically and genetically correlated with traditional reproductive traits (Darwash et al., 1997; Royal et al., 2002b; Berry et al., 2012). Furthermore, postpartum interval to the commencement of luteal activity is generally more heritable than traditional reproductive traits (Berry et al., 2014). This indicates that detailed reproductive traits may be less exposed to unrecorded management decisions (e.g., voluntary waiting periods), and thus a more pertinent assessment of the underlying reproductive performance of the cow.

The objective of the present study was to estimate genetic parameters for detailed reproductive traits derived from ultrasound examination of the reproductive tract in a large population of commercial dairy cows and to estimate the genetic correlations between these detailed reproductive traits and traditional reproductive traits. Results from our study will be useful in quantifying the genetic variation present in these detailed reproductive traits, but also their usefulness as part of a strategy to improve the accuracy and relevance of genetic evaluations for reproductive performance. Moreover, the results will be useful in elucidating the effect of current breeding strategies for traditional reproductive traits on the underlying detailed components of reproductive success.

\section{MATERIALS AND METHODS}

\section{Data}

Data from the Irish Cattle Breeding Federation database (http://www.icbf.com) were available on 5,872,465 calving events from $2,218,544$ dairy cows from 19,637 herds, as well as 4,299,285 insemination records from 1,255,632 dairy cows from 13,502 herds, between the years 2008 and 2013. Data were also available on 194,880 ultrasound records from 114,306 lactations on 72,120 dairy cows in 894 herds over the same period. Supplementary data on animal pedigree, breed composition, and cow parity were also available. Dairy breeds included Holstein-Friesian, Jersey, Montbeliarde, and Norwegian Red.

Traditional Reproductive Traits. Eight traditional reproductive traits were generated: (1) CI, (2) CFS, (3) DO, (4) number of inseminations (NI), (5) PRFS, (6) interval from first service to conception (IFC), (7) pregnant within $42 \mathrm{~d}$ of the herd breeding season (PR42), and (8) submission in the first $21 \mathrm{~d}$ of the herd breeding season (SR21). Calving interval was defined as the number of days between 2 consecutive calving events. Calving interval records were limited to be between 300 and $600 \mathrm{~d}$; if an insemination occurred within $150 \mathrm{~d}$ postpartum, CI was then restricted to between 300 and $800 \mathrm{~d}$. Calving to first service interval was defined as the number of days between calving and first insemination; only CFS records between 10 and $250 \mathrm{~d}$ were retained.

The approximate date of conception was determined as 283 d (i.e., average gestation length of different dairy breeds; Norman et al., 2009) before the subsequent calving date; where no subsequent calving date was available, date of conception was based on the reported gestational age of embryo or fetus at pregnancy diagnosis during ultrasound examination. The actual date of conception used in the present study was the last service date for that lactation, which was within $\pm 15 \mathrm{~d}$ of the estimated conception date previously described. If no corresponding insemination date was found within these margins, then conception date was set to missing. Days open was defined as the number of days between calving and conception; DO was limited to between 10 and $350 \mathrm{~d}$. Similarly, IFC was defined as the number of days between first service and the estimated date of conception, as described above.

Ireland operates a seasonal calving (and breeding) production system (Berry et al., 2013). The start of a herd's calving season in the present study was defined as the date when at least 5 cows within a herd calved within the subsequent 14-d period (Berry et al., 2013). A herd calving season was terminated on the date the last cow calved with no calving occurring in the subsequent $21 \mathrm{~d}$. Similarly, a herd's breeding season was initiated by the date when at least 5 cows were served within the subsequent $14-\mathrm{d}$ period and terminated by the last date a service occurred with no subsequent service occurring in the following $21 \mathrm{~d}$. Only data from calving seasons and breeding seasons between 35 and $100 \mathrm{~d}$ in length were retained.

The binary trait, SR21, was defined as whether a cow was inseminated in the first $21 \mathrm{~d}$ of the breeding season $(\mathrm{SR} 21=1)$ or not $(\mathrm{SR} 21=0)$; if a cow was submitted for insemination before the start of the breeding season, SR21 was set to missing. Number of services was defined as the number of inseminations within a lactation; number of services $>10$ were set to 10 . The binary trait, PR42, was defined as whether conception occurred $(\mathrm{PR} 42=1)$ or not $(\mathrm{PR} 42=0)$ in the first $42 \mathrm{~d}$ of the herd's breeding season; date of conception (previously described) was used in the calculation of PR42. If an insemination occurred or a cow was diagnosed as not pregnant after d 42 of the breeding season, failure 
to conceive within PR42 was assumed (i.e., PR42 = $0)$. Pregnancy rate to first service was determined as whether conception occurred $(\mathrm{PRFS}=1)$ or not (PFRS $=0$ ) to first service; date of conception was determined using the same approach already described. If a second insemination occurred, or a cow was diagnosed as not pregnant after first service, pregnancy was assumed not to have occurred to first service (i.e., PRFS $=0$ ). All data from herd-years with a pregnancy rate to first service of $>80 \%$ were discarded; this represented a small proportion $(3.5 \%)$ of herds that only recorded the last service per cow.

Detailed Reproductive Traits. Detailed reproductive traits were derived from ultrasound examination of the reproductive tract carried out by one company (Reprodoc Ltd., Fermoy, Co. Cork, Ireland) using real-time $\beta$-mode ultrasound scanner with a $5-\mathrm{MHz}$ transducer. The traits have been described in detail by both Carthy et al. (2014) and Fitzgerald et al. (2014a). The detailed reproductive traits included: (1) the resumption of cyclicity (CYC), (2) multiple ovulation (MO), (3) postpartum early ovulation (EO), (4) detected heat (DH), (5) cystic structures (CS), (6) embryo loss (EL), and (7) uterine score (US).

Resumption of cyclicity was defined as having resumed normal estrus cyclicity at the time of ultrasound examination and was defined in the present study as the presence $(\mathrm{CYC}=1)$ or the absence $(\mathrm{CYC}=0)$ of a non-cystic corpus luteum (CL) on the ovaries at the time of examination (Carthy et al., 2014). An MO was defined as the presence of $>1 \mathrm{CL}(\mathrm{MO}=1)$ on 1 or both ovaries at the time of examination in cycling cows (Fitzgerald et al., 2014a). Early ovulation was defined as ovulation having occurred $(\mathrm{EO}=1)$ or not $(\mathrm{EO}=0)$ by $15 \mathrm{~d}$ postpartum. Date of ovulation was determined by the size and density of the CL up to $6 \mathrm{~d}$ postovulation, after which an accurate ovulation date could not be determined. Therefore, EO could only be determined from ultrasound examinations up to $21 \mathrm{~d}$ postpartum; if examination occurred $>21 \mathrm{~d}$ postpartum, EO was set to missing.

Detected heat was only defined within a herd's AI breeding season. If no ovulation was detected by ultrasound examination during this period, $\mathrm{DH}$ was set to missing. If an insemination occurred within $5 \mathrm{~d}$ of the estimated date of ovulation, determined by ultrasound examination, then heat was assumed to have been detected $(\mathrm{DH}=1)$. If no recorded insemination existed within $5 \mathrm{~d}$ of the detected date of ovulation, heat was assumed not to have been detected $(\mathrm{DH}=0)$.

Cystic structures were defined as the presence (CS = 1 ) or absence $(\mathrm{CS}=0)$ of a $\mathrm{CS}(>25 \mathrm{~mm}$; follicular or luteal) on the ovaries at time of ultrasound examina- tion (Carthy et al., 2014). Uterine score, measured on a scale of 1 to 4, was based on the tone of the uterine wall, the size of the lumen, and the quantity of fluid present in the uterus (Carthy et al., 2014). Uterine score was described in nonpregnant cows by an assessment of the level of luminal fluid and tone in the uterine horns (Carthy et al., 2014). Four US were derived: (1) normal uterine tone with $<2 \mathrm{~mm}$ of luminal fluids; (2) normal uterine tone with 2 to $5 \mathrm{~mm}$ of luminal fluids; (3) poor uterine tone with 5 to $60 \mathrm{~mm}$ of luminal fluids; and (4) poor uterine tone with inflammation and $>60 \mathrm{~mm}$ of luminal fluids.

Embryo or fetal loss was assumed to have occurred if an embryo was deemed to be unviable at the time of ultrasound examination $(\mathrm{EL}=1)$. If, however, a subsequent calving date was less than $260 \mathrm{~d}$ from the date of pregnancy diagnosis $(<3 \%$ of recorded unviable embryos), EL was assumed not to have occurred (EL $=0$ ). Predicted calving date was calculated from the estimated gestational age of embryo or fetus at the time of examination, assuming a 283-d gestation length. If a cow was determined pregnant at ultrasound examination and the subsequent calving date was greater than $30 \mathrm{~d}$ after the predicted calving date then EL was assumed to have occurred $(\mathrm{EL}=1)$; if subsequent calving date was less than $30 \mathrm{~d}$ after the predicted calving date, then EL was assumed not to have occurred $(\mathrm{EL}=0)$. With the exception of an embryo determined unviable at ultrasound examination, if subsequent calving dates for a pregnancy diagnosis were not yet available (i.e., calving date is in the future) then EL was set to missing.

Data from cows with a CI $<300$ or $>600$ d were discarded. If no record of a subsequent calving date existed, cows calved $>600 \mathrm{~d}$ at the time of ultrasound examination and identified as nonpregnant were also discarded. After ultrasound examination, treatment may have been recommended for a selection of reproductive tract classifications. No knowledge, however, was available on whether or not any treatment subsequently occurred. Therefore, any ultrasound examinations for the remainder of that cow's lactation were discarded, with the exception of a pregnant diagnosis. Only herds that had more than $80 \%$ of their cows examined in a calendar year were retained.

\section{Data Editing}

Only animals with a known sire were retained. Parities 5 to 10 were grouped together as $5+$; parities $>10$ were discarded. Contemporary groups of herd-yearseason of calving were defined separately for each trait. The definition of herd-year-season was based on an 
Table 1. Number of records $(\mathrm{n})$, mean $(\mu)$, additive genetic SD $\left(\sigma_{\mathrm{g}}\right), \mathrm{h}^{2}$ (SE in parentheses) estimates, and repeatability across lactation (t; SE in parentheses) estimates for the traditional reproductive traits

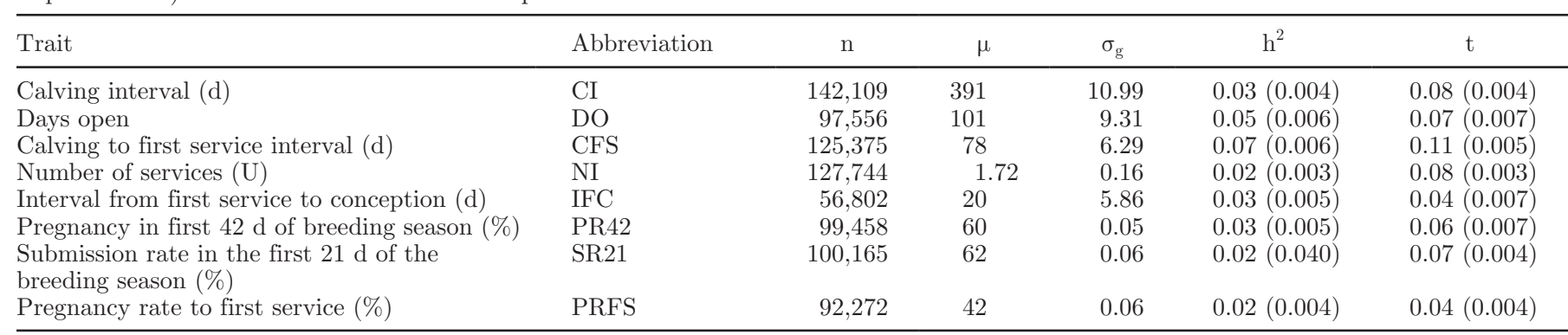

algorithm described in detail by Berry et al. (2013). Within a given herd, the algorithm groups animals together that calve around the same period of the year. Herd-year-seasons that contained fewer than 5 animals were discarded.

For computational reasons, a random sample of herds with traditional reproductive traits for each trait was taken; no such sampling was applied to the detailed reproductive traits. After all edits, up to 226,141 lactations with traditional reproductive measures from 96,020 cows in 2,927 herds, as well as 102,764 ultrasound records from 74,134 lactations on 47,750 cows in 658 herds remained; the number of records per trait are in Table 1 (traditional traits) and Table 2 (detailed traits). The pedigree of each animal was traced back at least 4 generations (where available). Coefficients of heterosis and recombination loss for each animal were calculated as outlined in VanRaden and Sanders (2003).

\section{Data Analysis}

Estimation of Genetic Parameters. Variance components were estimated by restricted maximum likelihood in ASREML (Gilmour et al., 2009) using a repeatability animal linear mixed model; univariate threshold models were also carried out for the binary traits. Genetic correlations between traits were estimated using a series of bivariate repeatability animal linear mixed models. The following models were used:

$$
\begin{gathered}
\mathrm{P}=\mu+\text { parity }+ \text { HYS }+ \text { DIM }+ \text { Het }+ \text { Rec } \\
+ \text { animal }+p e_{a}+p e_{w}+e, \text { and } \\
\mathrm{Q}=\mu+\text { parity }+ \text { HYS }+ \text { Het }+ \text { Rec } \\
+ \text { animal }+p e_{a}+e,
\end{gathered}
$$

where $\mathrm{P}$ is the observed trait of $\mathrm{CYC}$, CS, MO, US, and $\mathrm{DH}$ (i.e., potentially multiple measurements during lactation), Q is the observed trait of EL, EO, and all traditional reproductive traits (i.e., 1 measure per lactation), $\mu$ is the mean of trait, parity is the fixed effect of parity, HYS is the fixed effect of herd-year-season of calving, DIM is the fixed effect of a linear regression on DIM at the time of examination, Het is the fixed effect of heterosis, Rec is the fixed effect of recombination loss, animal is the random genetic effect, $p e_{a}$ is the random permanent environment effect across lactations, $p e_{w}$ is the random permanent environment effect within lactation, and $e$ is the random residual effect. Although, the genetic effect on EL may be separated into direct and maternal components, sire of the calf is only recorded when registering the calf after birth although the bull used in the insemination is sometimes recorded; insufficient data for EL remained when the data was restricted to when the sire of the embryo was known. Therefore, the direct genetic effect of the sire

\begin{tabular}{|c|c|c|c|c|c|c|}
\hline Trait & Abbreviation & $\mathrm{n}$ & $\mu$ & $\sigma_{\mathrm{g}}$ & $h^{2}$ & $\mathrm{t}$ \\
\hline Resumed of cyclicity (\%) & $\mathrm{CYC}$ & 49,587 & 87 & 0.08 & $0.07(0.009)$ & $0.31(0.007)$ \\
\hline Multiple ovulations (\%) & MO & 42,714 & 7 & 0.04 & $0.03(0.006)$ & $0.15(0.008)$ \\
\hline Early ovulation $^{1}(\%)$ & $\mathrm{EO}$ & 1,080 & 3 & 0.12 & $0.10(0.087)$ & \\
\hline Cystic structures (\%) & $\mathrm{CS}$ & 53,872 & 3 & 0.01 & $0.001(0.002)$ & $0.13(0.007)$ \\
\hline Uterine score (scale of 1 to 4 ) & US & 48,900 & 1.39 & 0.10 & $0.02(0.006)$ & $0.20(0.007)$ \\
\hline Embryo loss (\%) & EL & 43,473 & 8 & 0.04 & $0.02(0.006)$ & $0.66(0.005)$ \\
\hline
\end{tabular}
for EL was not estimable in the present study.

Table 2. Number of records $(\mathrm{n})$, mean $(\mu)$, additive genetic SD $\left(\sigma_{\mathrm{g}}\right), \mathrm{h}^{2}$ (SE in parentheses) estimates, and repeatability across lactation (t; SE in parentheses) estimates for the detailed reproductive traits

${ }^{1}$ No repeated records available to calculate repeatability. 
The covariance structure for the models was defined as

$$
\operatorname{var}\left[\begin{array}{c}
a \\
p e_{w} \\
p e_{a} \\
e
\end{array}\right]=\left[\begin{array}{cccc}
\mathbf{G} \otimes \mathbf{A} & 0 & 0 & 0 \\
0 & \mathbf{P}_{w} \otimes \mathbf{I} & 0 & 0 \\
0 & 0 & \mathbf{P}_{a} \otimes \mathbf{I} & 0 \\
0 & 0 & 0 & \mathbf{R} \otimes \mathbf{I}
\end{array}\right]
$$

where $\quad \mathbf{G}=\left[\begin{array}{cc}\sigma_{a_{1}}^{2} & \sigma_{a_{1} a_{2}} \\ \sigma_{a_{1} a_{2}} & \sigma_{a_{2}}^{2}\end{array}\right], \quad \mathbf{P}_{w}=\left[\begin{array}{cc}\sigma_{p e_{w 1}}^{2} & \sigma_{p e_{w 1} p e_{w 2}} \\ \sigma_{p e_{w 1} p e_{w 2}} & \sigma_{p e_{w 2}}^{2}\end{array}\right]$, $\mathbf{P}_{a}=\left[\begin{array}{cc}\sigma_{p e_{a 1}}^{2} & \sigma_{p e_{a 1} p e_{a 2}} \\ \sigma_{p e_{a 1} p e_{a 2}} & \sigma_{p e_{a 2}}^{2}\end{array}\right]$, and $\mathbf{R}=\left[\begin{array}{cc}\sigma_{e_{1}}^{2} & \sigma_{e_{1} e_{2}} \\ \sigma_{e_{1} e_{2}} & \sigma_{e_{2}}^{2}\end{array}\right] ; \mathbf{A}$ is numerator relationship matrix; I was an identity matrix of appropriate order; $\otimes$ is the Kronecker product; $\sigma_{a}^{2}, \sigma_{p e_{w}}^{2}, \sigma_{p e_{a}}^{2}$, and $\sigma_{e}^{2}$ are the additive genetic, permanent environment within lactation, permanent environment across lactation, and residual variance, respectively; and $\sigma_{a_{1} a_{2}}, \sigma_{p e_{w 1} p e_{w 2}}, \sigma_{p e_{a 1} p e_{a 2}}$, and $\sigma_{e_{1} e_{2}}$ are the additive genetic, permanent environment within lactation, permanent environment across lactation, and residual covariance components, respectively.

Selection Index Methodology. Alternative selection indexes were investigated to quantify the benefits of including detailed reproductive traits in the estimation of genetic merit for reproductive performance. The breeding objective for reproductive performance used was CI, the same trait used in the Irish national breeding index (Berry et al., 2007) and identical or similar (e.g., DO) to the reproductive traits included in most dairy cow national breeding objectives. The accuracy of the alternative selection indexes was calculated assuming different permutations of the number of progeny records available by different combinations of traits in the selection index. The main scenarios investigated include (1) using information from just CI, (2) using information from just CFS, (3) using information from all traditional traits, (4) using information from just CYC, (5) using information from all detailed traits and, (6) using a combination of CFS and CYC. The optimal index weights (b) were determined using $\mathbf{b}=\mathbf{P}^{-1} \mathbf{G a}$, where $\mathbf{P}^{-1}=$ the inverse of the phenotypic (co)variance matrix of the traits in the selection index, $\mathbf{G}=$ the genetic covariance matrix between traits in the selection goal and the selection index, and $\mathrm{a}=$ the vector containing the economic value for the goal trait; an economic value of 1 was assumed for the goal trait CI. The proportion of genetic variation in CI explained by the traits in the selection index was simplified as $\mathbf{C}^{\prime} \mathbf{V}^{-1} \mathbf{C}$, where $\mathbf{C}=$ the vector of genetic correlation(s) with $\mathrm{CI}$, and $\mathbf{V}=$ the genetic correlation matrix between traits in the selection index.

\section{RESULTS}

\section{Descriptive Statistics}

The mean CFS, DO, CI, and IFC were 76, 93, 385, and $6 \mathrm{~d}$, respectively (Table 1 ). Mean PRFS was 0.42 , whereas the mean PR42 was 0.60. The mean NI was 1.72 and the prevalence of $1,2,3$, and $>3$ inseminations was $57,26,10$, and $7 \%$, respectively. The mean percentage of cows that had resumed cyclicity at the time of ultrasound examination was $87 \%$ (mean DIM of 101 at the time of ultrasound examination), with $43 \%$ having resumed cyclicity by $15 \mathrm{~d}$ postpartum (Table 2 ). During the herd's breeding season, $90 \%$ of cows identified as having recently cycled had a corresponding insemination (i.e., $10 \%$ of ovulations detected by ultrasound did not have an associated insemination record). The prevalence of uterine score 2 (small quantity of fluid and normal uterine tone), 3 (large quantity of fluid and moderately flaccid uterine tone), and 4 (very large quantity of fluid with a flaccid tone) was 19.85 , 7.19 , and $1.51 \%$, respectively; this indicates that $8.7 \%$ of cows exhibited poor uterine environments (scores 3 and 4).

\section{Variance Components}

With the exception of CFS (0.07), the traditional reproductive traits were lowly heritable (i.e., 0.001 to 0.05; Table 1); repeatability (across parity) estimates ranged from 0.04 (PRFS) to 0.11 (CFS; Table 1). The heritability of the interval traits were greater than the binary and count traits. Heritability estimates for the detailed reproductive traits ranged from 0.001 (CS) to 0.10 (EO; Table 2); repeatability estimates ranged from 0.13 to 0.66 . Similar heritability estimates were obtained when a threshold model was applied (results not shown). The coefficient of genetic variation for the continuous traits CI, CFS, and DO was 0.03, 0.08, and 0.09 , respectively. The genetic standard deviations for the detailed binary traits ranged from 0.01 (CS) to 0.12 (EO). The detailed reproductive traits associated with cyclicity (i.e., CYC, EO, and DH) had the greatest genetic standard deviation (i.e., > 0.08).

Figure 1 illustrates paternal half-sib frequency distributions for CYC, CS, MO, and EL for sires with $>20$ daughters in $>10$ herds; too few sires remained to report these results for EO and DH. Half-sib mean prevalence per sire for resumed cyclicity by the time of examination varied from 65 and $98 \%$, with $14 \%$ of sires having a mean daughter prevalence of $>95 \%$. Paternal half-sib mean prevalence for embryo loss varied considerably from $3 \%$ of sires with no occurrence of embryo loss to over $21 \%$ of 1 sire's daughters experiencing embryo loss. 

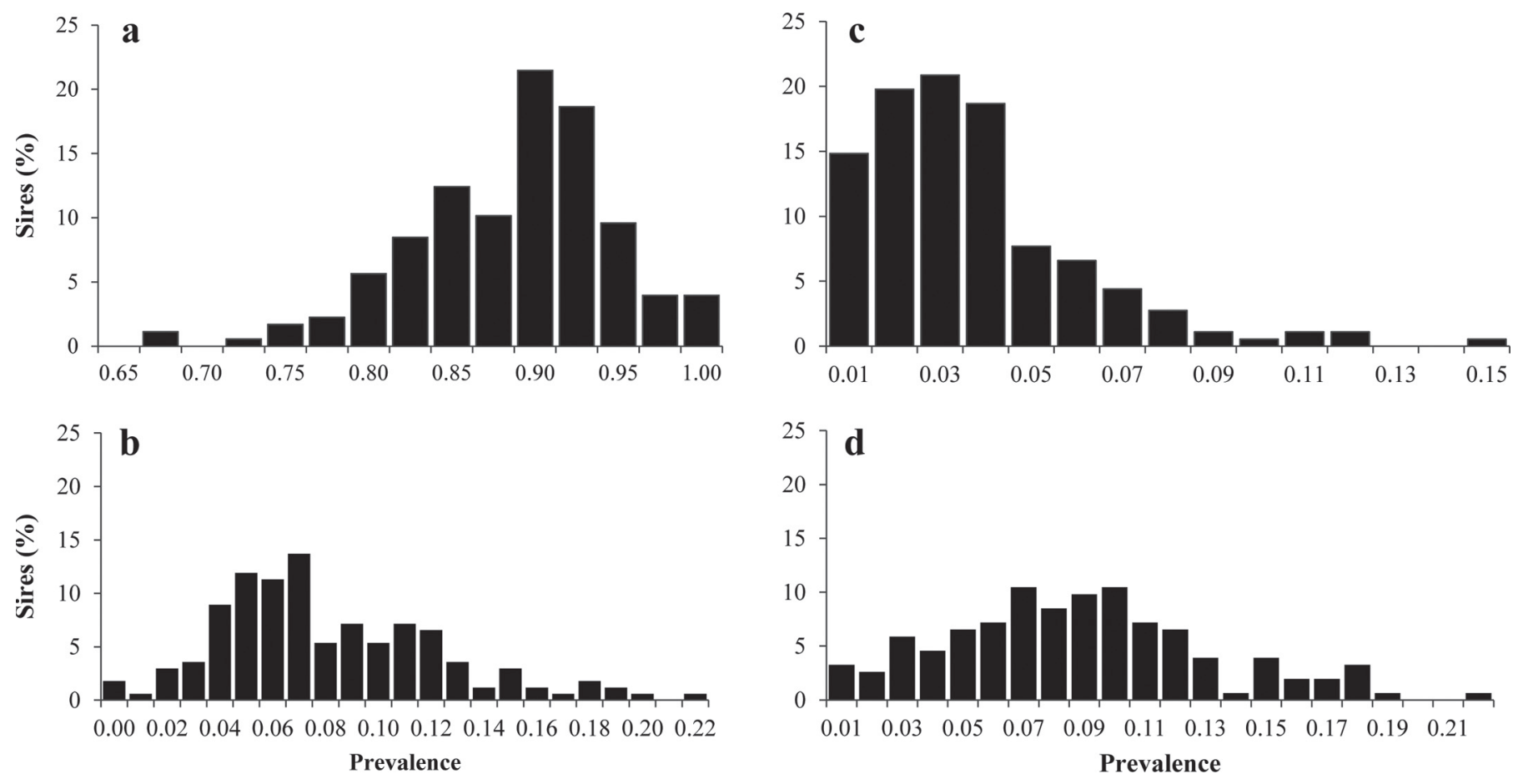

Figure 1. Half-sib family prevalence for (a) resumed cyclicity, (b) multiple ovulations, (c) cystic structures, and (d) embryo loss for sires with $>20$ daughters in $>10$ herds.

\section{Correlations Among the Traditional Reproductive Traits}

The genetic correlations among the traditional reproductive traits were generally stronger and of the same sign as the corresponding phenotypic correlations (Table 3). Inferior genetic merit for CI (i.e., larger values) was associated with inferior genetic merit for CFS, DO, IFC, and NI (genetic correlations between 0.60 and 0.99; Table 3), and a reduction (i.e., inferior) in genetic merit for SR21, PRFS, and PR42 (genetic correlations between -0.89 and -0.76 ; Table 3 ). The genetic correlations that existed between the remaining interval traits (i.e., CFS, IFC, and DO) and SR21, PRFS, PR42, and NI were comparable to the genetic correlations that existed with CI; longer intervals (i.e., CFS, DO, or IFC) were associated with poorer SR21 (with the exception of IFC), pregnancy rate, and increased NI (with the exception of CFS).

\section{Correlations Among the Detailed Reproductive Traits}

Moderate genetic and phenotypic correlations existed among some of the detailed reproductive traits (Table 4). Cows genetically predisposed to having resumed cyclicity by the time of ultrasound examination were genetically predisposed to an inferior US (genetic cor- relation of 0.29). No genetic correlation existed between resumed cyclicity and either CS or EL; however, a negative phenotypic correlation $(-0.18)$ existed between CYC and CS. Early ovulation was both genetically $(-0.92)$ and phenotypically $(-0.65)$ associated with a reduced incidence of CS. Genetic merit for inferior US was associated with greater genetic predisposition to MO and EL, whereas a worse US was also phenotypically associated with an increased likelihood of both $\mathrm{MO}$ and EL. Genetic predisposition to MO was associated with a greater genetic risk of EL (genetic correlation of 0.40 ). The remaining genetic correlations were not different from zero.

\section{Correlations Between the Traditional and Detailed Reproductive Trait}

Genetic correlations between the traditional reproductive traits and detailed reproductive traits are presented in Table 5 . The phenotypic correlations between the traditional and detailed reproductive traits were, in general, weaker than the corresponding genetic correlations (results not shown). The genetic correlations between CYC and the traditional reproductive traits were generally moderate to strong, ranging from -0.54 to -0.36 and from 0.54 to 0.70 , with the exception of those with NI and PRFS; cows with superior genetic 
merit for CYC had shorter intervals CFS, DO, and CI, but also had superior SR21 and PR42. Positive genetic correlations existed between US and all of CI, IFC, DO, and NI (0.18 to 0.52 ; Table 5$)$, whereas negative genetic correlations existed between US and both PR42 $(-0.32)$ and PRFS (-0.47); these correlations indicate that an inferior uterine environment was associated with reduced pregnancy rate, thereby extending the CI on average. Although standard errors were large, DH was genetically associated with shorter intervals to first service, conception, and subsequent calving, as well as improved SR21 and PR42. Embryo loss was genetically associated with poorer reproductive performance, such as longer CI, CFS, DO, and IFC (0.44 to 0.80), with poorer pregnancy rates $(-0.52$ to -0.42$)$. The genetic correlations between both CS and MO with the traditional traits were not different from zero.

\section{Selection Index}

Irrespective of the scenario investigated, the accuracy of selection for CI increased with the number of progeny records available (Figure 2). Assuming 100 progeny records were available, the accuracy of selection with CI phenotypic records alone (excluding information from pedigree) was 0.66 . The accuracy of selection using the different scenarios, assuming 100 progeny records were available, ranged from 0.42 (CYC alone) to 0.79 (CYC and CFS). There was a 9-percentage-unit increase in accuracy when all traditional reproductive traits were included, and a 3-percentage-unit increase when all detailed traits where included. A selection index that included CI as well as both cyclicity traits, CFS and CYC, achieved an 11-percentage-unit greater accuracy than an index based on CI alone. Calving to first service and CYC combined explained $52 \%$ of the genetic variation in $\mathrm{CI}$; when all traditional traits were included as well as cycle, the proportion of the genetic variation in CI explained increased to $89 \%$.

\section{DISCUSSION}

\section{Mean Phenotypic Performance}

Corroborating reported prevalence of cyclicity (89\%) from ultrasound examination on 7,797 Irish HolsteinFriesian cows not included in the present study (Mee et al., 2009), $87 \%$ of the cows in the present study had resumed cyclicity at the time of examination. Only $10 \%$ of the cycling cows in the present study, which were examined during the herd's AI breeding season, were not inseminated at ovulation. This could be an artifact of the producer's decision not to inseminate; however, as heat was only measured during the herd's 
Table 4. Genetic correlations (above the diagonal; SE in parenthesis) and phenotypic correlations (below the diagonal; SE in parenthesis) among the detailed reproductive traits $^{1}$

\begin{tabular}{|c|c|c|c|c|c|c|c|}
\hline Trait & CYC & MO & $\mathrm{EO}$ & $\mathrm{DH}$ & CS & US & EL \\
\hline $\mathrm{CYC}$ & & $-0.60(0.082)$ & $0.17(0.136)$ & $0.58(0.165)$ & $0.03(0.315)$ & $0.29(0.118)$ & $-0.08(0.171)$ \\
\hline MO & $-0.49(0.005)$ & & $-0.39(0.310)$ & $0.02(0.229)$ & $0.49(0.304)$ & $0.33(0.145)$ & $0.40(0.197)$ \\
\hline $\mathrm{EO}$ & $0.25(0.030)$ & $0.01(0.052)$ & & $0.21(0.480)$ & $-0.92(0.110)$ & $-0.05(0.269)$ & $0.25(0.411)$ \\
\hline $\mathrm{DH}$ & $0.09(0.036)$ & $0.04(0.029)$ & $-0.22(0.303)$ & & $0.42(0.543)$ & $0.10(0.224)$ & $0.05(0.107)$ \\
\hline $\mathrm{CS}$ & $-0.16(0.007)$ & $0.15(0.007)$ & $-0.65(0.023)$ & $-0.02(0.038)$ & & $-0.06(0.350)$ & $0.54(0.400)$ \\
\hline US & $0.02(0.005)$ & $0.00(0.005)$ & $-0.23(0.026)$ & $0.04(0.034)$ & $0.05(0.007)$ & & $0.24(0.200)$ \\
\hline EL & $0.04(0.013)$ & $0.02(0.014)$ & $-0.22(0.303)$ & $0.13(0.157)$ & $-0.03(0.014)$ & $0.28(0.012)$ & \\
\hline
\end{tabular}

${ }^{1} \mathrm{CYC}=$ resumption of cyclicity; $\mathrm{MO}=$ multiple ovulation; $\mathrm{EO}=$ early ovulation; $\mathrm{DH}=$ detected heat; CS $=$ cystic structures; US $=$ uterine score; EL = embryo loss.

AI breeding season in the present study (mean of $88 \mathrm{~d}$ with a minimum of $22 \mathrm{~d}$ postcalving for the animals not inseminated), it was assumed that any cow detected in heat would be inseminated; inseminating cows detected in estrus is the norm in Ireland (and other seasonal calving herds), especially if calved several months.

The prevalence of CS (3\%) and MO (7\%) was lower than the respective statistics reported internationally (Hooijer et al., 2001; Zwald et al., 2004; López-Gatius et al., 2005; Stevenson et al., 2007), whereas the prevalence of EL was consistent with previously reported prevalence both nationally (7.2\%; Silke et al., 2002) and internationally (8.1\%; Silva-Del-Río et al., 2009). Fitzgerald et al. (2014b) documented a greater prevalence of CS and MO in higher-yielding cows, providing an explanation for the lower prevalence in the present study, as milk production in grazing dairy cows is expected to be less than cows fed TMR diets.

\section{Variance Components}

Heritability of the traditional reproductive traits in the present study was low, corroborating the consensus of low heritability estimates reported internationally for reproductive performance in dairy cows (Berry et al., 2014). Of particular note was the fact that return to cyclicity postcalving was most heritable, irrespective of whether the definition was CFS or the detailed traits of $\mathrm{EO}$ or CYC; this is also consistent with a meta-analysis of 28 studies that reported variance components for different reproductive traits in dairy and beef cattle (Berry et al., 2014). The observed heritability of return to cyclicity in the present study was lower than the heritability estimate for postpartum commencement of luteal activity reported previously in dairy cows (Darwash et al., 1997; Royal et al., 2002b; Berry et al., 2012). Differences between studies may be due to the data set in the present study being collected from field data, which may be experiencing greater environmental effects (and error) compared with the previous studies from controlled experiments.

Heritability estimates for both EO and DH, although larger than the other reproductive traits, were also associated with large standard errors attributable mainly to the smaller data set available for these traits. Early ovulation was defined within the first $15 \mathrm{~d}$ postpartum, and to our knowledge no heritability estimates for this period have been previously reported. Nonetheless, the heritability estimates obtained for EO were similar to estimates obtained for other traits related to cyclicity, both in the present and other studies (Berry et al., 2014). Detected heat was defined as a failure to inseminate at ovulation; a negative outcome for this trait may have resulted from silent heat, a producer not detect-

Table 5. Genetic correlations (SE in parenthesis) between the detailed reproductive traits and the traditional reproductive traits ${ }^{1}$

\begin{tabular}{|c|c|c|c|c|c|c|c|}
\hline Trait & $\mathrm{CYC}$ & $\mathrm{MO}$ & $\mathrm{EO}$ & $\mathrm{DH}$ & CS & US & EL \\
\hline DO & $-0.56(0.092)$ & $0.17(0.133)$ & $-0.22(0.330)$ & $-0.24(0.200)$ & $-0.14(0.300)$ & $0.18(0.137)$ & $0.44(0.141)$ \\
\hline CFS & $-0.59(0.069)$ & $0.12(0.111)$ & $0.41(0.251)$ & $-0.33(0.132)$ & $0.15(0.245)$ & $-0.09(0.113)$ & $0.55(0.165)$ \\
\hline IFC & $-0.36(0.121)$ & $0.14(0.155)$ & $-0.26(0.403)$ & $-0.24(0.231)$ & $-0.37(0.326)$ & $0.30(0.154)$ & $0.67(0.173)$ \\
\hline PR42 & $0.54(0.128)$ & $-0.06(0.101)$ & $0.15(0.401)$ & $0.33(0.251)$ & $0.14(0.356)$ & $-0.32(0.162)$ & $-0.42(0.218)$ \\
\hline SR21 & $0.70(0.084)$ & $-0.07(0.140)$ & $0.42(0.355)$ & $0.36(0.213)$ & $0.34(0.314)$ & $0.09(0.144)$ & $-0.17(0.188)$ \\
\hline
\end{tabular}

${ }^{1} \mathrm{CYC}=$ resumption of cyclicity; $\mathrm{MO}=$ multiple ovulation; $\mathrm{EO}=$ early ovulation; $\mathrm{DH}=$ detected heat; $\mathrm{CS}=$ cystic structures; US = uterine score; $\mathrm{EL}=$ embryo loss; $\mathrm{CI}=$ calving interval; $\mathrm{DO}=$ days open; $\mathrm{CFS}=$ calving to first service interval; $\mathrm{NS}=$ number of services; $\mathrm{IFC}=$ interval from first service to conception; PR42 = pregnancy in first $42 \mathrm{~d}$ of breeding season;; SR21 = submission rate in the first $21 \mathrm{~d}$ of the breeding season; PRFS = pregnancy rate to first service. 


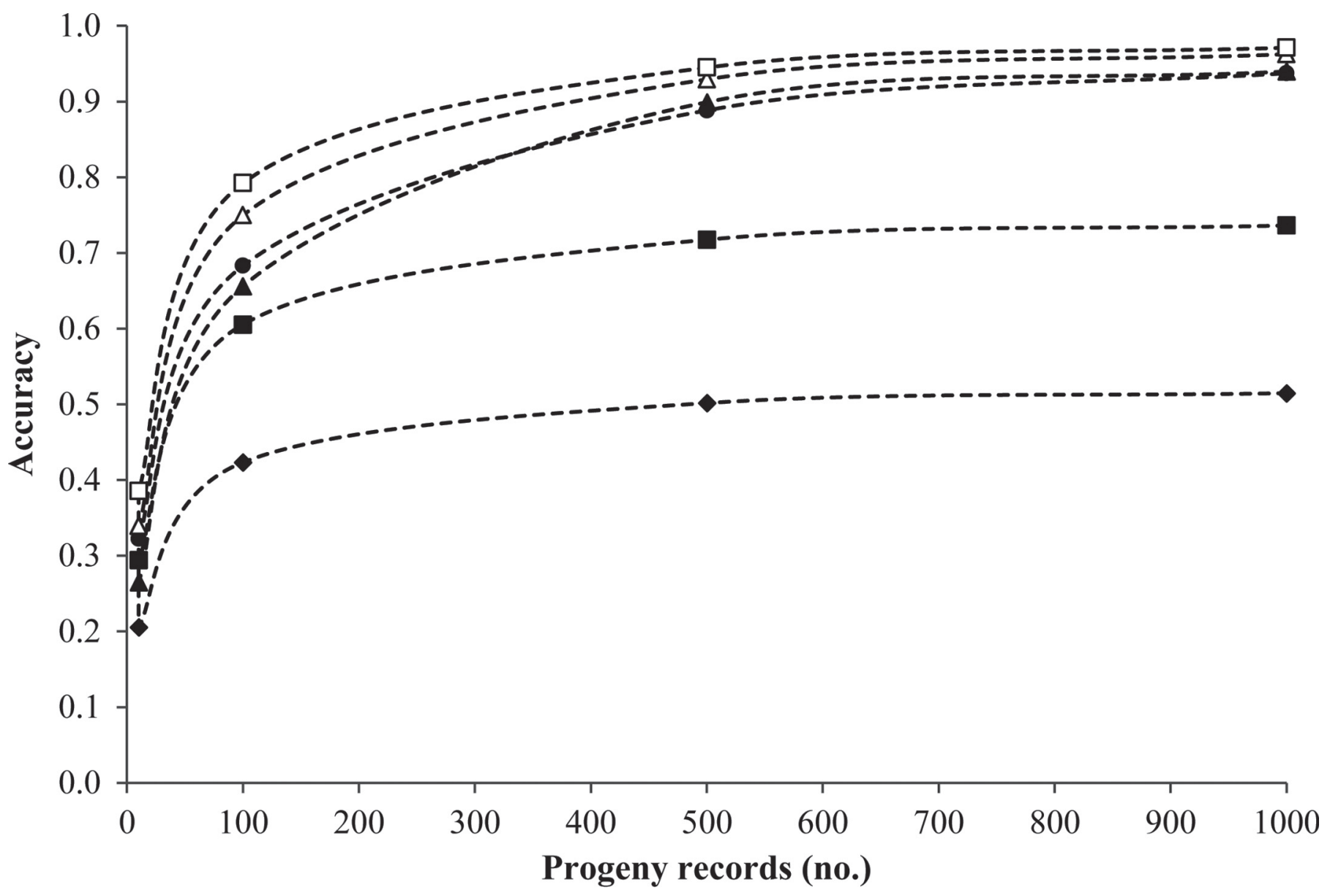

Figure 2. The accuracy of prediction for genetic merit for calving interval with increasing number of progeny records, using (1) calving interval $(\boldsymbol{\Lambda})$, (2) resumed cyclicity $(\diamond)$, (3) calving to first service $(\boldsymbol{\square})$, (4) resumed cyclicity and calving to first service ( $\square$ ), (5) all detailed reproductive traits $(\bullet)$, and $(6)$ all traditional reproductive traits $(\Delta)$.

ing the heat, or a conscious decision not to inseminate. The genetic variation in this trait suggests that factors other than simply a random management decision not to inseminate a cow in estrus are likely contributing to this. Genetic components of heat expression have been documented for intensity of heat (Roxström et al., 2001), duration of heat (Løvendahl and Chagunda, 2009), and silent heat (Heringstad, 2010; Koeck et al., 2010; Gernand et al., 2012).

Genetic parameters for MO in a subset of the present study have been reported and discussed in detail elsewhere (Fitzgerald et al., 2014a). Previous studies documented greater heritability estimates for ovarian cystic structures than observed in the present study, with documented ranges varying from 0.02 to 0.09 in United States (Zwald et al., 2004), Dutch (Hooijer et al., 2001), and, more recently, Canadian (Koeck et al., 2014) dairy cow populations; however, the prevalence of ovarian cystic structures was also greater in these populations (ranging from 7.4 to $8.2 \%$ ). Even after converting the heritability from the underlying scale to a observed scale (Dempster and Lerner, 1950), the heritability for cystic structures in the present study (0.01) remained lower than in other studies (Hooijer et al., 2001; Zwald et al., 2004; Koeck et al., 2014).

Heritability estimates for uterine infections vary considerably across different populations and breeds, from 0.005 in Finish Ayrshire cows (Pösö and Mäntysaari, 1996) to 0.07 in United States Holstein cows (Zwald et al., 2004); the heritability obtained in the present study for US was within this international range. Heritability estimates for the different assessments of uterine infections (acute metritis, purulent discharge, endometritis, and chronic metritis) are similar to each other and the genetic correlations among these traits were not different from unity (Koeck et al., 2012), suggesting they are genetically similar traits; thus, one holistic measure of overall uterine infection is plausible (Koeck et al., 
2012); therefore, a trait such as US may be a feasible way to measure uterine infection.

Bamber et al. (2009) previously documented a heritability estimate for embryo loss (0.16), primarily in Holstein cows, greater than that obtained in the present study, albeit with larger associated standard errors. In the present study, EL was identified anytime between $21 \mathrm{~d}$ postinsemination and the end of gestation, whereas Bamber et al. (2009) only considered pregnancy loss between 30 and $60 \mathrm{~d}$ postinsemination, on average. The more definitive period of embryo loss previously reported could have less associated environmental variation, thereby resulting in a greater heritability estimate.

Despite the low heritability estimates observed for all the reproductive traits in the present study, considerable exploitable additive genetic variation did exist, as evident by the coefficient of genetic variation and the genetic standard deviations of both traditional and detailed reproductive traits. This is further substantiated by the existence of considerable variation in prevalence of the detailed traits among paternal half-sib groups (Figure 1). Of note was the sire family that had the greatest prevalence of embryo loss ( $21 \%$ embryo loss based on 38 daughters in 10 herds); this sire was among the worst $2 \%$ in the Irish national genetic evaluations for CI in the August 2014 genetic evaluation. High prevalence of embryo loss among paternal half-sib families could be indicative of carriers of the lethal recessive haplotypes (VanRaden et al., 2011) or other harmful genetic mutations.

\section{Correlations Among the Reproductive Traits}

The observed phenotypic and genetic correlations among the traditional reproductive traits are similar to those previously reported nationally on a subset of these data (Berry et al., 2013) as well as documented in the meta-analysis of international studies (Berry et al., 2014). Despite just under $90 \%$ of the genetic variation in CI explained by the other traditional reproductive traits, $10 \%$ of the genetic variation remains unexplained.

Contrary to what may be expected, the association between CYC and US in the present study indicated that genetic merit for increased (i.e., superior) cyclicity was associated with inferior genetic merit for uterine environment (i.e., higher uterine score). Early resumption of cyclicity is generally accepted as beneficial to reproductive performance, but progesterone levels associated with estrous cyclicity may inhibit the ability of the uterus to eliminate bacteria and return to normal postpartum (Heppelmann et al., 2013). Therefore, despite the favorable phenotypic association between uterine environment and cyclicity, an unfavorable genetic association exists and needs to be considered when selecting on the ability to resume cyclicity postpartum using such traits as CFS or CYC. Furthermore, the uterine environment is important in establishing and maintaining pregnancy; an inferior uterine environment postpartum was genetically associated with a greater likelihood of embryo loss. The results of the present study, therefore, indicate that not all the detailed aspects of reproduction performance are favorably associated with each other.

The genetic correlations between the detailed reproductive traits and traditional reproductive traits, nonetheless, indicate that, despite the unfavorable genetic correlation between resumed cyclicity and uterine score, superior genetic merit for the traditional reproductive traits was associated with favorable outcomes in both of these traits. Inferior uterine environment postpartum has been shown to be genetically associated with poorer reproductive performance (Pösö and Mäntysaari, 1996). This corroborates the results of the present study, where a 1-unit increase in genetic merit for uterine score (i.e., inferior uterine score) was associated with a genetically longer CI by $26 \mathrm{~d}$. Early resumption of cyclicity postpartum is, nonetheless, an important aspect of reproductive performance, which was reflected in the genetic association between cyclicity and the traditional reproductive traits. Interval to commencement of luteal activity postpartum, measured by progesterone levels, has been shown to be genetically associated with CI (Royal et al., 2002a; Berry et al., 2012), further corroborating the genetic correlations observed in the present study between CI and the ability to resume cyclicity postpartum.

Reproductive traits in heifers and lactating cows have been shown to be genetically different traits (Tiezzi et al., 2012); therefore, excluding heifer reproductive performance may bias genetic evaluation. As ultrasound examinations were performed on commercial herds where heifers are rarely presented for ultrasound examination before pregnancy diagnosis and AI is not routinely used unless as part of a synchronization program, it was not possible to accurately identify detailed reproductive traits in heifers. Further research on heifer reproductive performance may provide a better understanding of the relationship between detailed reproductive traits in heifers and other reproductive traits.

\section{Selection Index}

The goal trait used in in the current study, as well as many national evaluations, CI, is producer-recorded and can be affected by unrecorded management deci- 
sion, such as voluntary waiting period and preferential treatment of high-producing cows as well as inaccurate recording; these could contribute to greater residual error. Furthermore, CI phenotypes are only available once a subsequent calving event occurs; this can lead to a long waiting period to obtain the phenotype, but will also exclude animals that fail to subsequently calve. However, despite its limitation, CI is easy to collect on large scale, as only calving dates are required and bias in favor of animals that fail to recalve can be accounted for through the inclusion of survival in a multitrait evaluation. The greater heritability of traits associated with cyclicity and the strong correlations that exist between these traits and CI suggest that these traits could be important predictor traits of reproductive performance in a multitrait genetic evaluation. Based on the (co)variance components estimated in the present study, a 1 percentage unit improvement in genetic merit for CYC was associated with a shorter genetic merit for CI by $0.72 \mathrm{~d}$. Using selection index methodology, greater accuracy was achieved for relatively small progeny group size when traits related to cyclicity were included in the selection index than when selecting using information on CI alone. Furthermore, based on the correlations obtained in the present study, the 2 cyclicity traits, CFS and CYC, not only improved accuracy, but explained over half (i.e., $52 \%$ ) of the genetic variation in CI.

\section{CONCLUSIONS}

Detailed aspects of reproductive performance have been shown to exhibit heritable genetic variation as well as being correlated with traditional measures of reproductive performance. Selection on the complex phenotypes of CI (or similar traits, such as DO), as is practiced in most breeding programs, is improving the detailed attributes of reproductive performance considered in the present study. Reproductive traits related to ability to resume cyclicity postpartum were the most heritable of the reproductive traits and were strongly correlated with the aggregate reproductive traits (CI and DO). Furthermore, although some of these detailed traits have economic values in their own right (i.e., requirement of veterinary intervention), they were also shown to be useful predictors of genetic merit for CI.

\section{ACKNOWLEDGMENTS}

Funding from the Department of Agriculture, Food and Marine Research Stimulus Fund (RSF 11/S/133; Dublin, Ireland) and the OptiMIR project (http:// www.optiMIR.eu) is gratefully acknowledged.

\section{REFERENCES}

Bamber, R. L., G. E. Shook, M. C. Wiltbank, J. E. P. Santos, and P. M. Fricke. 2009. Genetic parameters for anovulation and pregnancy loss in dairy cattle. J. Dairy Sci. 92:5739-5753.

Berry, D. P., J. Bastiaansen, R. Veerkamp, S. Wijga, E. Wall, B. Berglund, and M. Calus. 2012. Genome-wide associations for fertility traits in Holstein-Friesian dairy cows using data from experimental research herds in four European countries. Animal 6:1206-1215.

Berry, D., J. Kearney, K. Twomey, and R. Evans. 2013. Genetics of reproductive performance in seasonal calving dairy cattle production systems. Ir. J. Agric. Food Res. 52:1-16.

Berry, D., L. Shalloo, A. Cromie, R. Veerkamp, P. Dillion, P. Amer, J. Kearney, R. Evans, and B. Wickham. 2007. The economic breeding index: A generation on. Pages 29-34 in Technical Report to the Irish Cattle Breeding Federation. Co. Cork, Ireland. www.icbf. com.

Berry, D. P., E. Wall, and J. E. Pryce. 2014. Genetics and genomics of reproductive performance in dairy and beef cattle. Animal $8: 105-121$.

Carthy, T. R., D. P. Berry, A. Fitzgerald, S. McParland, E. J. Williams, S. T. Butler, A. R. Cromie, and D. Ryan. 2014. Risk factors associated with detailed reproductive phenotypes in dairy and beef cows. Animal 8:695-703.

Darwash, A. O., G. E. Lamming, and J. A. Woolliams. 1997. Estimation of genetic variation in the interval from calving to postpartum ovulation of dairy cows. J. Dairy Sci. 80:1227-1234.

Dempster, E. R., and I. M. Lerner. 1950. Heritability of threshold characters. Genetics 35:212-236.

Fitzgerald, A. M., D. Berry, T. Carthy, A. Cromie, and D. Ryan. 2014a. Risk factors associated with multiple ovulation and twin birth rate in Irish dairy and beef cattle. J. Anim. Sci. 92:966-973.

Fitzgerald, A. M., D. P. Ryan, T. R. Carthy, R. D. Evans, and D. P. Berry. 2014b. Ovarian structures and uterine environment are associated with phenotypic and genetic merit for performance in lactating dairy cows. Theriogenology 82:1231-1240.

Gernand, E., P. Rehbein, U. U. von Borstel, and S. König. 2012. Incidences of and genetic parameters for mastitis, claw disorders, and common health traits recorded in dairy cattle contract herds. J. Dairy Sci. 95:2144-2156.

Gilmour, A., B. Cullis, S. Welham, and R. Thompson. 2009. ASREML Reference Manual. New South Wales Agriculture, Orange Agricultural Institute, Orange, NSW, Australia.

Heppelmann, M., A. Brömmling, M. Weinert, M. Piechotta, C. Wrenzycki, and H. Bollwein. 2013. Effect of postpartum suppression of ovulation on uterine involution in dairy cows. Theriogenology $80: 519-525$

Heringstad, B. 2010. Genetic analysis of fertility-related diseases and disorders in Norwegian Red cows. J. Dairy Sci. 93:2751-2756.

Hooijer, G. A., R. B. F. Lubbers, B. J. Ducro, J. A. M. van Arendonk, L. M. T. E. Kaal-Lansbergen, and T. van der Lende. 2001. Genetic parameters for cystic ovarian disease in dutch black and white dairy cattle. J. Dairy Sci. 84:286-291.

Koeck, A., C. Egger-Danner, C. Fuerst, W. Obritzhauser, and B. Fuerst-Waltl. 2010. Genetic analysis of reproductive disorders and their relationship to fertility and milk yield in Austrian Fleckvieh dual-purpose cows. J. Dairy Sci. 93:2185-2194.

Koeck, A., S. Loker, F. Miglior, D. F. Kelton, J. Jamrozik, and F. S. Schenkel. 2014. Genetic relationships of clinical mastitis, cystic ovaries, and lameness with milk yield and somatic cell score in first-lactation Canadian Holsteins. J. Dairy Sci. 97:5806-5813.

Koeck, A., F. Miglior, D. F. Kelton, and F. S. Schenkel. 2012. Health recording in Canadian Holsteins: Data and genetic parameters. J. Dairy Sci. 95:4099-4108.

López-Gatius, F., M. López-Béjar, M. Fenech, and R. Hunter. 2005. Ovulation failure and double ovulation in dairy cattle: risk factors and effects. Theriogenology 63:1298-1307.

Løvendahl, P., and M. Chagunda. 2009. Short communication: Genetic variation in estrus activity traits. J. Dairy Sci. 92:4683-4688.

Mee, J. F., F. Buckley, D. Ryan, and P. Dillon. 2009. Pre-breeding ovaro-uterine ultrasonography and its relationship with first service 
pregnancy rate in seasonal-calving dairy herds. Reprod. Domest. Anim. 44:331-337.

Miglior, F., B. Muir, and B. Van Doormaal. 2005. Selection indices in Holstein cattle of various countries. J. Dairy Sci. 88:1255-1263.

Norman, H. D., J. R. Wright, M. T. Kuhn, S. M. Hubbard, J. B. Cole, and P. M. VanRaden. 2009. Genetic and environmental factors that affect gestation length in dairy cattle. J. Dairy Sci. 92:2259-2269.

Pösö, J., and E. A. Mäntysaari. 1996. Genetic relationships between reproductive disorders, operational days open and milk yield. Livest. Prod. Sci. 46:41-48.

Roxström, A., E. Strandberg, B. Berglund, U. Emanuelson, and J. Philipsson. 2001. Genetic and environmental correlations among female fertility traits, and between the ability to show oestrus and milk production in dairy cattle. Acta Agric. Scand. A Anim. Sci. $51: 192-199$.

Royal, M. D., J. Pryce, J. Woolliams, and A. Flint. 2002a. The genetic relationship between commencement of luteal activity and calving interval, body condition score, production, and linear type traits in Holstein-Friesian dairy cattle. J. Dairy Sci. 85:3071-3080.

Royal, M. D., A. P. F. Flint, and J. A. Woolliams. 2002b. Genetic and phenotypic relationships among endocrine and traditional fertility traits and production traits in Holstein-Friesian dairy cows. J. Dairy Sci. 85:958-967.
Silke, V., M. G. Diskin, D. A. Kenny, M. P. Boland, P. Dillon, J. F. Mee, and J. M. Sreenan. 2002. Extent, pattern and factors associated with late embryonic loss in dairy cows. Anim. Reprod. Sci. $71: 1-12$.

Silva-Del-Río, N., J. D. Colloton, and P. M. Fricke. 2009. Factors affecting pregnancy loss for single and twin pregnancies in a highproducing dairy herd. Theriogenology 71:1462-1471.

Stevenson, J. S., M. Portaluppi, and D. Tenhouse. 2007. Factors influencing upfront single-and multiple-ovulation incidence, progesterone, and luteolysis before a timed insemination resynchronization protocol. J. Dairy Sci. 90:5542-5551.

Tiezzi, F., C. Maltecca, A. Cecchinato, M. Penasa, and G. Bittante. 2012. Genetic parameters for fertility of dairy heifers and cows at different parities and relationships with production traits in first lactation. J. Dairy Sci. 95:7355-7362.

VanRaden, P. M., K. Olson, D. Null, and J. Hutchison. 2011. Harmful recessive effects on fertility detected by absence of homozygous haplotypes. J. Dairy Sci. 94:6153-6161.

VanRaden, P. M., and A. H. Sanders. 2003. Economic merit of crossbred and purebred US dairy cattle. J. Dairy Sci. 86:1036-1044.

Zwald, N. R., K. A. Weigel, Y. M. Chang, R. D. Welper, and J. S. Clay. 2004. Genetic selection for health traits using producerrecorded data. I. Incidence rates, heritability estimates, and sire breeding values. J. Dairy Sci. 87:4287-4294. 